\title{
Excitonic condensation in quasi-two-dimensional systems
}

\author{
M. Crisan \\ Department of Theoretical Physics, University of Cluj, 400084 Cluj-Napoca, Romania
}

I. Ţifrea

Department of Theoretical Physics, University of Cluj, 400084 Cluj-Napoca, Romania and

Department of Physics and Astronomy, The University of Iowa, Iowa City, IA 52242, USA

\begin{abstract}
We present a low energy model for the Bose-Einstein condensation in a quasi-two-dimensional excitonic gas. Using the flow equations of the Renormalization group and a $\Phi^{4}$ model with the dynamical critical exponent $z=2$ we calculate the temperature dependence of the critical density, coherence length, magnetic susceptibility, and specific heat. The model can be relevant for the macroscopic coherence observed in GaAs/AlGaAs coupled quantum wells.
\end{abstract}

\section{INTRODUCTION}

The Bose-Einstein condensation (BEC) in interacting bosonic systems has attracted increasing attention in the recent years due to the practical realization of a condensate state in trapped alkali-metal atoms. Also, a sustained experimental effort was directed to the realization and visualization of the BEC of excitons in low dimensional systems, namely in semiconductor quantum wells (QW). 1, 2] A remarkable property of the BEC state is that a large number of particles, which are in the condensate state, become dependent on a single wave function promoting quantum properties to classical length and time scale. This state can be described by a wave function with a phase coherence over distances much larger than separation between individual particles. Along with BEC states in pure bosonic systems such as alkali atoms, there are a series of BEC states formed from composite bosons. For example, such a state appears in superconductors, where the Cooper pairs can be treated as composite bosons formed from two electrons which interact via an attractive potential. In standard superconductors the average characteristic length of the Cooper pairs (the coherence length) is much larger than the actual distance between pairs, making the BEC state a state of overlapping Cooper pairs. This situation is in contrast with the case of a BEC state in pure bosonic systems such as helium and atomic vapors of metals, where the bosons are single particles and the system can be treated in a dilute limit.

The BEC state in semiconductors is obtained from the condensation of excitons, which are also composite bosons formed from an electron bounded to a hole. Excitons are usually created by shining light on the semiconductor, as a result an equal number of electrons and holes being created. The electrons interact with the holes leading to the formation of electron-hole $(\mathrm{e}-\mathrm{h})$ bound states. Theoretically the formation of excitons was predicted to appear in semiconductors 3] or metals. 4 In metallic systems such bound states lead to the itinerant electron antiferromagnetism studied initially in the mean field approximation. [5, 6, 7] On the other hand, in semiconductor bulk systems, the short life time of optically generated excitons was a major obstacle to the experimental study of such a system. The recent advances in the realization of low dimensional semiconductor systems such as QW made possible to overcome the issues of a short life time in bulk systems. Semiconductor QW allow the confinement of electrons and holes in layered configurations, which can be assimilated to quasi-two dimensional (2D) systems.

A slightly different situation occurs in the case of bilayer QW where indirect excitons can be formed between the conduction band electrons from one layer and the valence band holes from the adjacent layer. The spatial separation between electrons and holes give rise to a repulsive interaction between the excitons, which prevent the occurrence of the e-h plasma. Experimental studies of the bilayer QW system showed an enhance exciton mobility, an increased radiative decay rate, and a photoluminescence noise. All these results are considered to be an indirect argument for the BEC of the excitons as they are connected to an increased coherence among the system. A detailed and clear discussion of the particular features of the BEC in bosonic systems formed by fermions (Cooper pairs and excitons) was given a long time ago by Kohn and Sherrington. [8] The critical behavior and the role of excitonic fluctuations have been investigated using the renormalization group (RNG) method, $[9]$ with the main result being the evidence of a dimensional crossover between a semi-metallic and a semiconducting state in three dimensional (3D) systems. Such a crossover effect is determined by the band overlap and is influenced by the presence of non-magnetic impurities in the system.

Here, we will study using the RNG the possibility of a BEC of excitons in systems with a reduced dimensionality. In such systems, which usually are considered to be quasi-2D systems, the BEC is known as a quasicondensation because it does not appear in the thermodynamic limit. The model we explore is similar to the one applied to a 2D bosonic system, 10] used to address the physical properties of the condensed state in He. Based on this model we investigate the thermodynamic properties of the system as the temperature dependence of the critical density, coherence length, magnetic susceptibility, and specific heat. 


\section{RENORMALIZATION GROUP APPROACH}

The bosonic system formed by the excitons can be described by the following general action:

$$
S=\frac{1}{2} \int d \tau d^{d} \mathbf{r}\left[\Phi^{*} \partial_{\tau} \Phi-\left(\nabla^{2}-\mu\right)|\Phi|^{2}+\frac{t_{0}}{8}|\Phi|^{4}\right],
$$

where $\Phi \equiv \Phi(\mathbf{r}, \tau)$ is the bosonic field describing the $e-h$ density fluctuations, $t_{0}$ is the bare interaction between the bosons and $\mu$ is the effective chemical potential. In the case of an excitonic gas formed in a semiconductor system the effective chemical potential is defined as:

$$
\mu(n)=\varepsilon_{g}-\varepsilon_{0},
$$

where $\varepsilon_{g}$ is the semiconducting band gap and $\varepsilon_{0}$ is the exciton binding energy. The spatial scale of the problem, for which the interaction between the component excitons treated as bosons becomes relevant, is determined by the smallest length in the system, namely, the radius of the exciton, $r_{0} \sim \varepsilon_{0}^{1 / 2}$. The existence of a low density parameter is determined by the range $r_{0}$ of the interaction potential, $t_{0}$, and the mean separation between the constituent particles, $\bar{l}\left(\mu \sim 1 /\left(\bar{l}^{2}\right)\right)$. In this case

$$
r_{0} \sqrt{\mu} \ll 1 \text {. }
$$

Under this condition we can describe the condensation of the excitons in a large temperature interval, including the critical region.

To address the phase transition in the excitonic gas we used the method introduced by Popov 11] to describe the standard Bose gas. Accordingly, we introduce an intermediate momentum $p_{i}$ such that $\mu<\varepsilon_{i}<\varepsilon_{0}$, with $\varepsilon_{i}=p_{i}^{2} /(2 m)$. The effective interaction between the constituen particles, $u_{0}$, is obtained in the t-matrix approximation as:

$$
u_{0}=\frac{t_{0}}{1+t_{0} \Pi}
$$

In Eq. (4) the polarization, $\Pi$, is given by:

$$
\Pi=\int_{1 / r_{0}}^{p_{i}} \frac{d^{d} k}{(2 \pi)^{d}} \frac{1}{k^{2}} .
$$

The integration in Eq. (5) is strongly dependent on the system dimensionality. For the case of a two-dimensional system, $d=2$, the effective interaction can be approximated as

$$
u_{0} \simeq \frac{2}{\pi \ln \frac{\varepsilon_{0}}{\varepsilon_{i}}}
$$

a value which according to the energy scale of the problem is small. On the other hand, for $d>2$, the effective interaction can be calculated as $u_{0} \simeq C r_{0}^{(d-2)}$, where $C$ is a constant. For the two dimensional case, the polarization is logarithmical small, and the resulting effective interaction is repulsive and small. As a result we can use the RNG formalism, applied to a modified action, to describe the Bose gas formed by the constituent excitons. The resulting action may describe a quantum phase transition (QPT) in the two dimensional exciton Bose system at finite temperature. The formalism is similar to that developed in Refs. 10, 12 for the weakly interacting Bose system.

The standard Wilson RNG procedure, leads to the following set of differential equations corresponding to the renormalized chemical potential, $\mu(l)$, interaction, $u(l)$, and temperature, $T(l)$ :

$$
\begin{gathered}
\frac{d \mu(l)}{d l}=2 \mu(l)+K_{2} F_{1}(r, T) u(l), \\
\frac{d u(l)}{d l}=-\frac{K_{2}}{4} u(l)^{2},
\end{gathered}
$$

and

$$
\frac{d T(l)}{d l}=2 T(l)
$$

Here $K_{2}=1 /(2 \pi)$ and $F_{1}(\mu, T)$ is given by:

$$
F_{1}(\mu, T)=\frac{1}{2} \operatorname{coth} \frac{1}{2 T(l)} .
$$

This set of differential equations can be solved starting from the last equation to the first one. From Eqs. (9) and (8), we get

$$
T(l)=T \exp (2 l)
$$

and

$$
u(l)=\frac{1}{C\left(l+l_{0}\right)}
$$

with $C=K_{2} / 4$ and $l_{0}=8 \pi / u_{0}$. Following the same methods as in Refs. 10, 12 we calculated the renormalized chemical potential, $\mu(l)$, as:

$$
\begin{aligned}
\mu(l)= & \exp [\Lambda(l)]\left[\mu-\mu_{c}-\frac{1}{2 \pi} \int_{0}^{l} \frac{d l^{\prime} e^{-2 l^{\prime}} u\left(l^{\prime}\right)}{e^{1 / T\left(l^{\prime}\right)}-1}\right] \\
& -\frac{u(l)}{8 \pi}
\end{aligned}
$$

where $\mu_{c}=u_{0} /(8 \pi)$ and $\Lambda(l)$ is given by the expression:

$$
\Lambda(l)=2 l\left[1-\frac{1}{l} \ln \left(1+\frac{l}{l_{0}}\right)\right] .
$$

The possibility of a phase transition in the excitonic system can be studied if we introduce a new parameter, $t_{\mu}(l)$, defined as:

$$
t_{\mu}(l)=\mu(l)+\frac{u(l)}{8 \pi}
$$


Based on Eqs. (12) and (13), $t_{\mu}(l)$ can be written as:

$$
t_{\mu}(l)=\exp [\Lambda(l)] t_{\mu}(T),
$$

where

$$
t_{\mu}(T)=\left[t_{\mu}(0)+\frac{u_{0} T}{4 \pi} \int_{a}^{\infty} \frac{d x}{\exp [x]-1}\right],
$$

with $a=u_{0} /(4 \pi)$. The renormalization procedure will be stopped at $l=l^{*}$, where $l^{*}$ is the solution of the following equation:

$$
t_{\mu}\left(l^{\star}\right)=1 .
$$

From Eqs. (15) and (16) we find $l^{\star}$ as:

$$
\exp \left(-2 l^{\star}\right) \cong \frac{T}{\ln (1 / T)},
$$

a value which in the following will be used to study the main thermodynamic properties of the excitonic system.

\section{THERMODYNAMICS IN THE CRITICAL REGION}

The RNG permits the evaluation of the main thermodynamical properties of the system in the critical region around the QPT point. The correlation length, $\xi$, and the magnetic susceptibility, $\chi$, can be obtained using the procedure presented in Refs. [10, 12] as:

$$
\xi\left(\mu, u_{0}, T\right) \simeq \xi_{0} \exp \left(l^{\star}\right)
$$

and:

$$
\chi\left(\mu, u_{0}, T\right) \simeq \chi_{0} \exp \left(2 l^{\star}\right),
$$

where $\xi_{0}$ and $\chi_{0}$ are constants and the value for $l^{\star}$ given by the Eq. (19). A simple calculation leads to:

$$
\xi(T) \simeq \xi_{0} \frac{|\ln (1 / T)|^{1 / 2}}{T^{1 / 2}}
$$

and:

$$
\chi(T) \simeq \chi_{0} \frac{|\ln (1 / T)|}{T} .
$$

The critical density, $n(T)$, will be calculated using the renormalized Bose-Einstein function considered in $l=l^{*}$ :

$$
n(T)=\exp \left(-2 l^{\star}\right) \int_{0}^{\infty} \frac{d^{2} k}{(2 \pi)^{2}}\left\{\exp \left[\frac{k^{2}+\mu}{T\left(l^{*}\right)}\right]-1\right\}^{-1}
$$

In the case of a two-dimensional system the integral can be calculated analytically leading to

$$
n(T) \simeq T \ln |\ln (1 / T)| .
$$

This equation can be inverted to calculate the critical temperature for the BEC in the excitonic system [10]:

$$
T_{c}(n) \simeq \frac{n}{\ln |\ln (1 / n)|} .
$$

Another important parameter of the critical region is the specific heat. In order to obtain its temperature dependence we consider the scaling of the free energy, $f(l)$, written as [10]:

$$
\frac{d f(l)}{d l}=4 f(l)+g[\mu(l), T(l)],
$$

where :

$$
g(l) \simeq \frac{K_{2}}{2} \int_{0}^{l} d l^{\prime} \exp \left(-4 l^{\prime}\right)\left[1+\mu\left(l^{\prime}\right)\right] .
$$

The specific heat in the critical region will be calculated using the standard definition, $C(T)=-T \partial^{2} f(T) / \partial T^{2}$, using for the chemical potential the renormalized value obtained from Eq. (13). A simple calculation leads to a temperature dependence whose most divergent contribution is given by:

$$
C(T) \simeq \frac{T}{|\ln T|^{3}},
$$

a result similar to that obtained for the two-dimensional Bose system [10].

\section{CONCLUSION}

In this paper we developed a formalism based on the RNG approach for bosonic systems in order to describe the physical properties of an excitonic gas. We consider the case of a two dimensional system, a configuration which is very close to the quasi two dimensional geometries of GaAs quantum wells, where an excitonic condensation can be observed. Our result shows that in the case of a two dimensional system the critical temperature for the BEC in the excitonic system has a double logarithmical dependence on the density. Similar studies [13] showed a different dependence of the critical temperature. However, the models considered in Ref. 13. are three dimensional models with an in-plane harmonic or square potential confinement, which cannot reproduce the exact result from the two dimensional calculations.

The many-body methods for the calculation of the optical absorbtion-gain spectra has been formulated in Ref. 14] at finite temperatures. The authors claim that fluctuations cannot destroy the condensation of excitons even at finite temperature. The effects of exciton BEC can be observed up to $130 \mathrm{~K}$, which is a very high temperature. However, it is well known that the mean-field approximations overestimate the critical temperature, but the results are important because they show how to include the short-range Coulomb potential with screening in the problem. 
Here we proposed a microscopic description of the excitonic condensation in a two dimensional $(d=2)$ system in terms of an effective action similar to that for the interacting Bose liquid with repulsive short-range interaction. For this model the effective coupling constant between the component excitons was evaluated using a t-matrix approximation, and based on the characteristic energy scale we showed that it is small. Accordingly, the RNG method in the one-loop approximation can give relevant results. The critical density presents a non-linear temperature dependence, which can describe the behav- ior of the experimental results in a relevant temperature interval. A similar non-linear dependence was observed experimentally 1]. The critical temperature, compared to the expression obtained in Ref. [13] and used in Ref. 1] to explain the experimental behavior is much smaller. A more realistic model, has to consider the influence of trapping in the RNG formalism for the two dimensional system, but such a calculation is much more complicated. However, even this oversimplified model showed the importance of the quantum effects in the condensation of the excitons.
[1] L.V. Butov, A. Zrenner, G. Abstreiter, G. Bohm, and G. Weimann, Phys. Rev. Lett. 73, 304 (1994); L. V. Butov and A. I. Filin, Phys. Rev. B58, 1980 (1998); L. V. Butov, A. C. Gossard, and D. S. Chemla, Nature 418,751 (2002).

[2] D. Snoke, S. Denev, Y. Liu, L. Pfeiffer, and K. West, Nature 418, 754 (2002).

[3] L. V. Keldysh and A. N. Kozlov, Zh. Exp. Teor. Fiz. 54, 978 (1968) [Sov. Phys. JETP 27, 521 (1968)].

[4] A. Overhauser, Phys. Rev. B 128 (1970)

[5] P. A. Fedders and P. C. Martin, Phys. Rev. 143, 245 (1966).

[6] T. M. Rice, Phys. Rev. B 2, 2619 (1970).

[7] M. Crisan, Phys. Rev. B 9, 4838 (1974).

[8] W. Kohn and D. Sherrington, Rev. Mod. Phys. 42 1,
(1970).

[9] Y. Baba, T. Nagai, and K. Kawasaki, J. Low Temp. Phys. 36, 1 (1979).

[10] M. Crisan, D. Bodea, I. Grosu, and I. Tifrea, J. Phys. A: Math. Gen. 35, 239 (2002).

[11] V.N. Popov, Functional Integrals in Quantum Field Theory and Statistical Physics, Cambridge University Press, Cambridge (1987).

[12] A. Caramico D'Auria, L. De Cesare, and I. Rabuffo, Physica A 327, 442 (2003).

[13] W. Ketterle and N. J. van Druten, Phys. Rev. A 54, 656 (1996).

[14] H. Chu and Y.C. Chang, Phys. Rev. B54, 5020 (1996). 